\title{
Single session per oral endoscopic myotomy and trans oral incisionless fundoplication - can we prevent reflux in patients with achalasia? $\square$
}

\section{(ㄷ)(우우}

\section{Authors}

Petros C. Benias ${ }^{1}$, Vivek Kumbhari², Nikhil A. Kumta ${ }^{3}$, Amrita Sethi ${ }^{4}$, Lionel S. D’Souza ${ }^{5}$, Amy Tyberg ${ }^{6}$, Olaya Brewer Gutierrez ${ }^{2}$, Larry S. Miller ${ }^{1}$, David L. Carr-Locke ${ }^{7}$, Mouen A. Khashab ${ }^{2}$

Institutions

1 Division of Gastroenterology, Zucker School of Medicine at Hofstra/Northwell, Northwell Health System, Manhasset, New York, United States

2 Division of Gastroenterology and Hepatology, Johns Hopkins Medical Institutions, Baltimore, MD, United States.

3 Division of Gastroenterology and Hepatology, Mount Sinai, New York, New York, United States.

4 Division of Gastroenterology, Columbia University Medical Center, New York, New York.

5 Division of Gastroenterology and hepatology, Stony Brook School of Medicine, East Setauket, New York, United States

6 Department of Gastroenterology, Rutgers Robert Wood Johnson University Hospital, New Brunswick, New Jersey, United States.

7 Division of Gastroenterology and Hepatology, Weil Cornell School of Medicine, New York Hospital, New York, New York, United States

submitted 18.9.2020

accepted after revision 12.11 .2020

\section{Bibliography}

Endosc Int Open 2021; 09: E828-E835

DOI 10.1055/a-1395-5667

ISSN 2364-3722

(c) 2021. The Author(s).

This is an open access article published by Thieme under the terms of the Creative Commons Attribution-NonDerivative-NonCommercial License, permitting copying and reproduction so long as the original work is given appropriate credit. Contents may not be used for commercial purposes, or adapted, remixed, transformed or built upon. (https://creativecommons.org/licenses/by-nc-nd/4.0/)

Georg Thieme Verlag KG, Rüdigerstraße 14,

70469 Stuttgart, Germany

Corresponding author

Petros C. Benias, Hofstra Northwell School of medicine -

Division of Gastroenterology, 300 Community Drive,
Manhassett New York 11030, USA

Fax: +1-516.387.3990

pbenias@gmail.com

pbenias@northwell.edu

\section{ABSTRACT}

Background and study aims The rate of gastroesophageal reflux disease (GERD) after per oral endoscopic myotomy (POEM) is concerning. Endoscopic anti-reflux methods, such as Trans Oral Incisionless Fundoplication (TIF), are crucial for the long-term success of POEM, especially if they can be performed in the same session.

Methods We completed a proof-of-concept canine pilot study $(n=6)$ to assess safety and feasibility of POEM and TIF in a single session (POEM-TIF). Subsequently, POEM-TIF was also performed in patients with achalasia $(n=5)$. Herein, we report on the safety, technical and clinical success of the first-in-human cases with symptom follow-up at 1, 3 and 6 months and $\mathrm{pH}$ testing at 6 months.

Results POEM was completed successfully in six canines ( 3 anterior and 3 posterior myotomies), followed by TIF in the same session. Necropsy and extensive testing demonstrated no evidence of mucosal injury and no leaks. The reconstructed valve was 220 to 240 degrees, 3 to $4 \mathrm{~cm}$ in length, and resulted in concomitant esophageal lengthening (2-5 $\mathrm{cm})$. Using similar principles, the first-in-human cases were performed without intraprocedural or delayed adverse events. $\mathrm{pH}$ testing at 6 months showed that four of five patients had no evidence of GERD (DeMeester > 14.72), and in one case, there was evidence of esophagitis.

Conclusions Single session POEM-TIF appears to be safe and feasible. Early clinical human data suggests that it may be able to reduce post POEM GERD, however the additional secondary benefits such as lengthening and straightening of the esophagus, may prove to be equally important for the long-term success of POEM. 


\section{Introduction}

Peroral endoscopic myotomy (POEM) has been accepted worldwide as a minimally invasive endoscopic treatment for achalasia with results comparable to laparoscopic Heller myotomy (LHM). It has a low adverse event rate of $5-10 \%[1,2]$, with thousands performed worldwide. Despite success, concerns continue to arise over post-POEM gastroesophageal reflux disease (GERD), as this is considered significant with no obvious solution.

It has been suggested that POEM preserves peri-esophageal anti-reflux barriers, thereby not disrupting anatomical anti-reflux mechanisms. However, in large POEM series GERD is estimated to occur 40-60\% [3-5]. Concerns are increasing regarding GERD after POEM [6], with reports of esophagitis, Barrett's Esophagus, and even adenocarcinoma [7]. There is also a concern that GERD may increase over time affecting the long-term durability [6].

Kumbhari et al. assessed GERD after POEM showing that $58 \%$ of patients had objective evidence of abnormal acid exposure, with $23 \%$ showing some esophagitis. More concerning was that $60 \%$ of patients were asymptomatic [3]. This highlights the issue that many patients do not report symptoms, either related to the silent nature of GERD or the nature of achalasia to develop an insensate esophagus.

For LHM, the importance of adding a hemi-fundoplication was established early and is considered crucial to the long-term success of the procedure $[5,8]$. A large meta-analysis by Campos et al showed that in 7855 patients, the addition of an antireflux procedure to LHM reduced GERD from $31.5 \%$ to $8.8 \%$ [5]. On the other hand, POEM has been associated with higher rates of symptoms and evidence of esophagitis as compared to LHM $[4,9]$.

It is imperative to investigate endoscopic solutions for GERD that can be performed in the same session as POEM, allowing the procedure to mimic its surgical counterpart. To date, Transoral Incisionless Fundoplication (TIF) has emerged as the endoscopic gold standard for GERD [10-13] with long-term data on safety and durability [13]. Recently, TIF following POEM was reported in small select cohorts as a separate procedure $[14,15]$. We performed a canine pilot study to assess the safety and feasibility of performing POEM and TIF in the same session, while also investigating the ability of TIF to create an anti-reflux flapvalve as well as lengthening of the esophagus. We also report the results of the first in human POEM-TIF case series.

\section{Methods}

\section{Canine model}

A canine model was used to perform POEM-TIF due its prior success for TIF training. Six adult dogs (average weight $21.25 \pm 0.84$ $\mathrm{kg}$ ) underwent general anesthesia with propofol $(5 \mathrm{mg} / \mathrm{kg})$, and acepromazine $(0.1 \mathrm{mg} / \mathrm{kg})$. Procedures were non-survival, followed by necropsy and resection of an en bloc esophagogastroduodenal specimen for extensive leak testing. This protocol was approved by the local IACUC committee of Temple University.

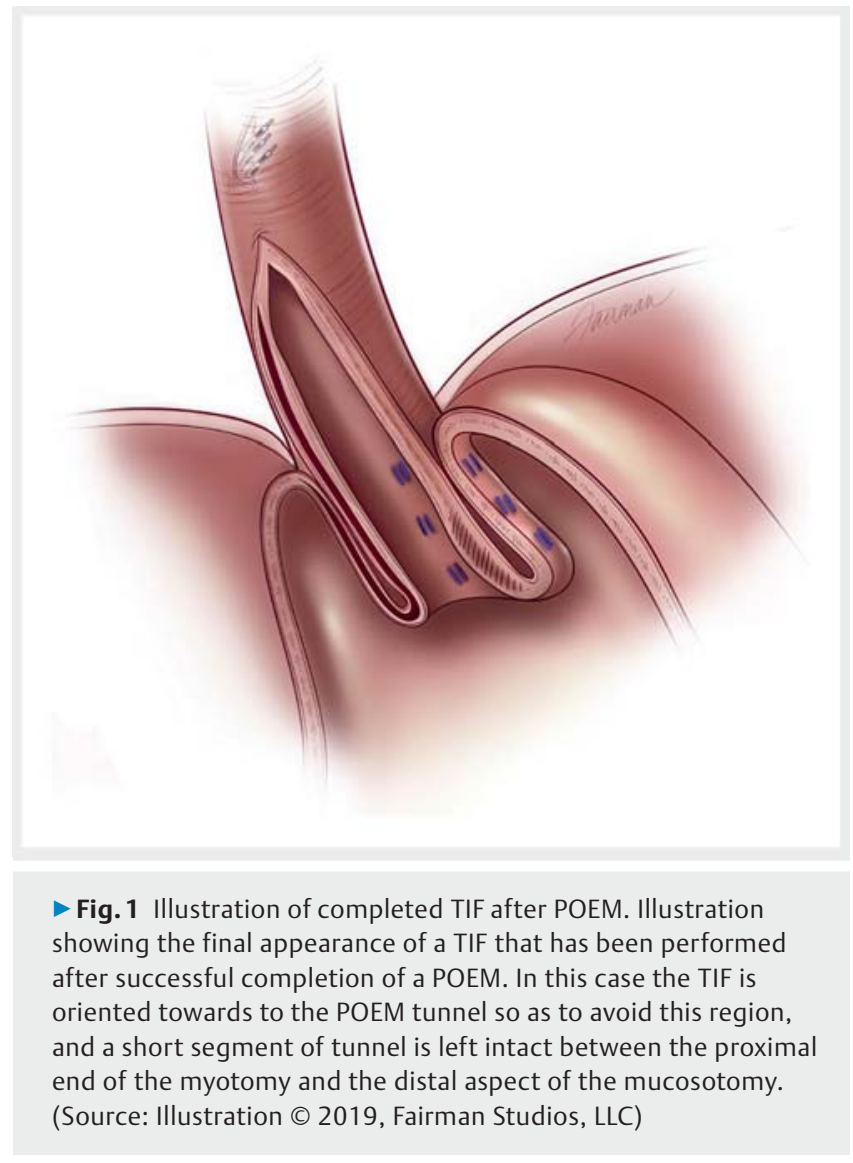

\section{POEM-TIF procedure}

POEM was performed by experienced operators (>100 POEMs individually; PCB, AS, NK, LSD, VK, DCL, LM) with the intention of performing a subsequent TIF ( $>$ Fig. 1). Procedures were performed with a gastroscope and transparent hood (GIF-Q180; Olympus, Tokyo, Japan). Regular air insufflation was used for the first case due to lack of $\mathrm{CO} 2$ insufflators. Subsequently, all procedures were performed using $\mathrm{CO} 2$ insufflation (ERBE ECO2, Tubingen, Germany).

POEM was performed in an anterior or posterior right orientation. A mucosotomy was initiated $10 \mathrm{~cm}$ proximally to the gastroesophageal junction (GEJ) ( $\mathbf{F i g . 1 , ~}>$ Fig.2a). The submucosal tunnel was extended 2 to $3 \mathrm{~cm}$ beyond the GEJ followed by a full thickness myotomy (Hybrid knife T-Type knife; ERBE, Tubingen, Germany) ( Fig.2b). After the myotomy, location of the POEM tunnel was demarcated with a soft coagulation mark (ERBE Soft Coagulation, 50 watts) placed on the gastric mucosa, $1 \mathrm{~cm}$ distal to the tunnel. This helped orient the subsequent TIF ( $\mathbf{F i g . 2 c}$ ). Closure of the entry was deferred until completion of the TIF, allowing inspection of the tunnel for bleeding and inadvertent injuries.

After completion of the POEM and inspection of the tunnel, a TIF was performed using the EsophyX Z + device (Endogastric Solutions, Redmond, Washington). Using the soft coagulation mark as the 12 o'clock reference position, a standard 240 to 270 fundoplication with approximately 20 fasteners was performed ( $>$ Fig.2e, $>$ Fig.3). After completion, and before clo- 

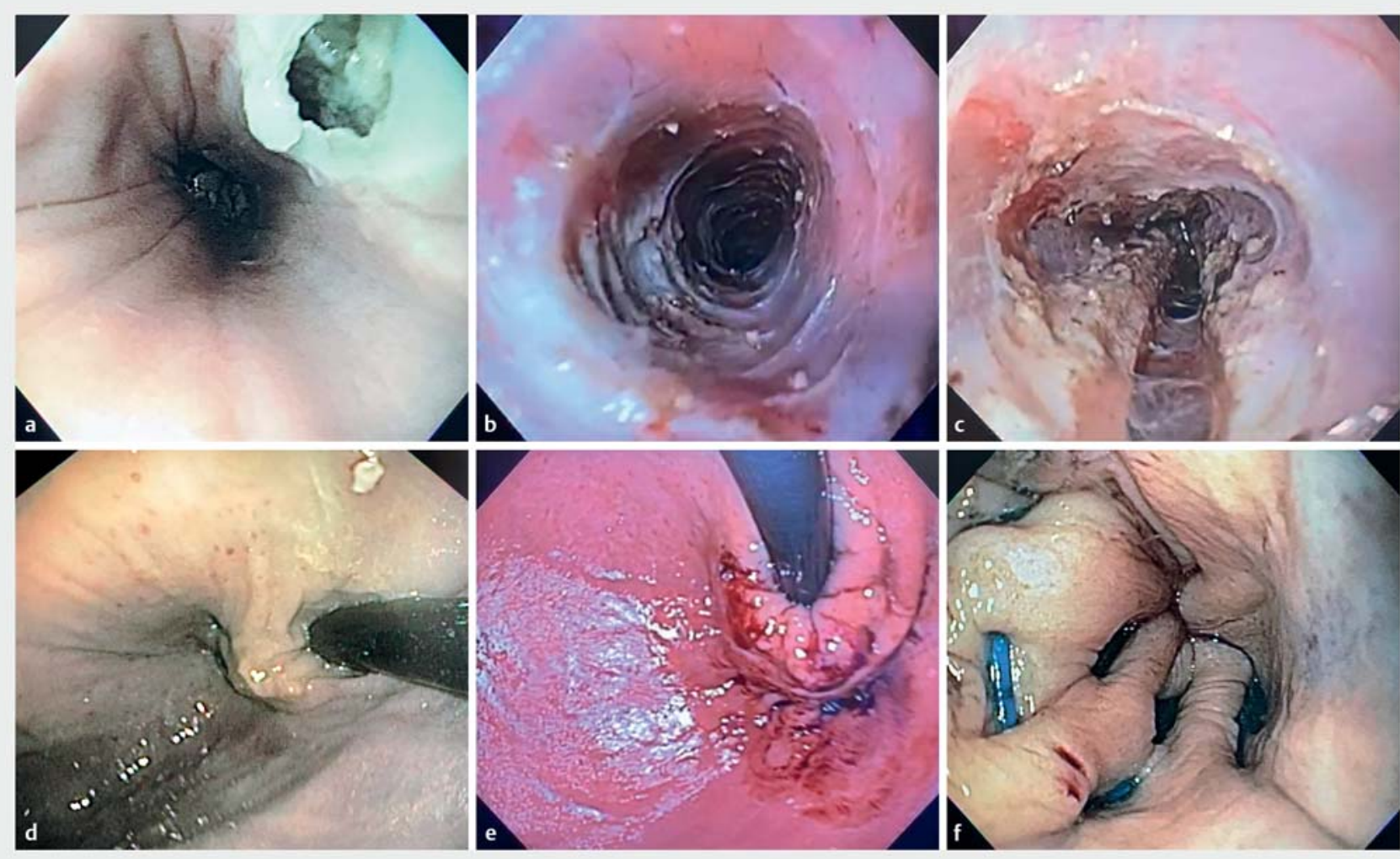

- Fig. 2 Composite showing various steps. a A small longitudinal mucosotomy is created, and $\mathbf{b}$ in a standard fashion a submucosal tunnel is extended along the anterior right aspect of the esophagus. c The POEM is completed, and a soft coagulation mark placed along the axis of the tunnel on the gastric side, $\mathbf{d}$, e so that a TIF can be safely performed while orienting the fundoplication to the myotomy. $\mathbf{f}$ The tunnel is inspected again before final closure showing no bleeding or damage to the overlying mucosa.
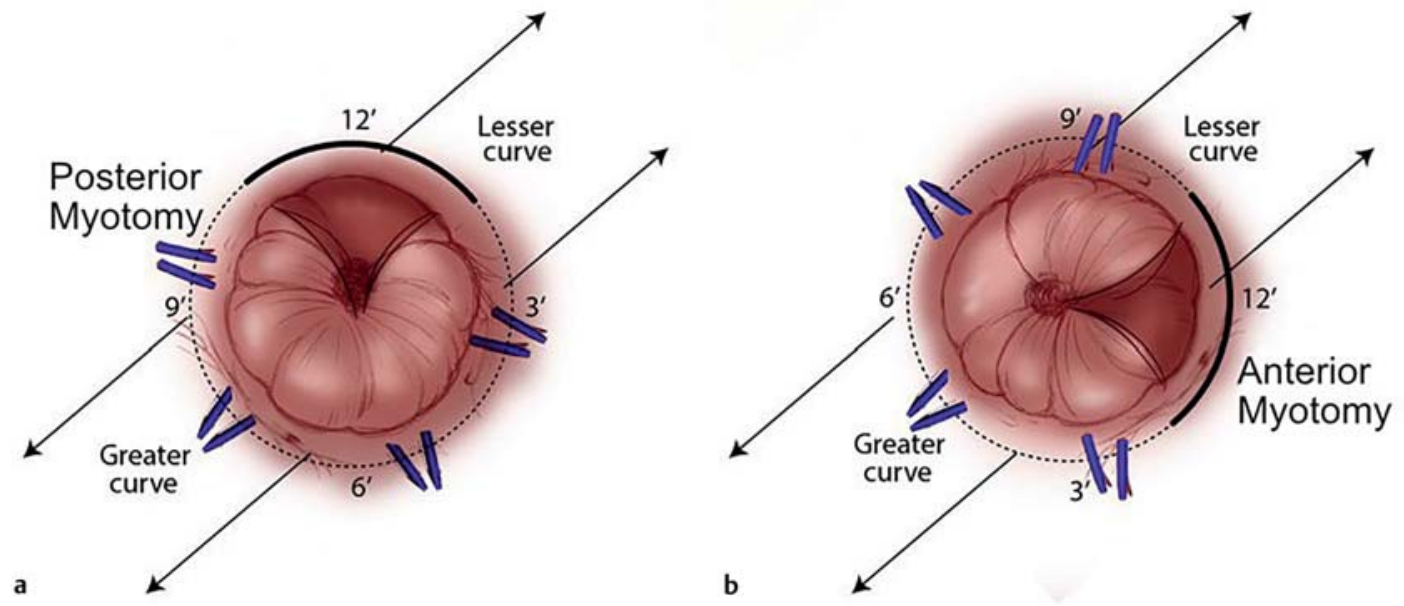

Posterior retroflex views of $270^{\circ}$ plication

Fig. 3 Illustration showing the concept of how TIF can be oriented to an anterior or posterior POEM tunnel Illustration of a retroflexed view of a TIF performed after a a posterior myotomy and also $\mathbf{b}$ an anterior myotomy. Typically, TIF is oriented to the lesser curvature, but in TIFPOEM a small adjustment allows us to orient the fundoplication to the POEM (12 o'clock), which is slightly anterior or just slightly posterior to the lesser curvature. (Source: Illustration ( $) 2019$, Fairman Studios, LLC) 


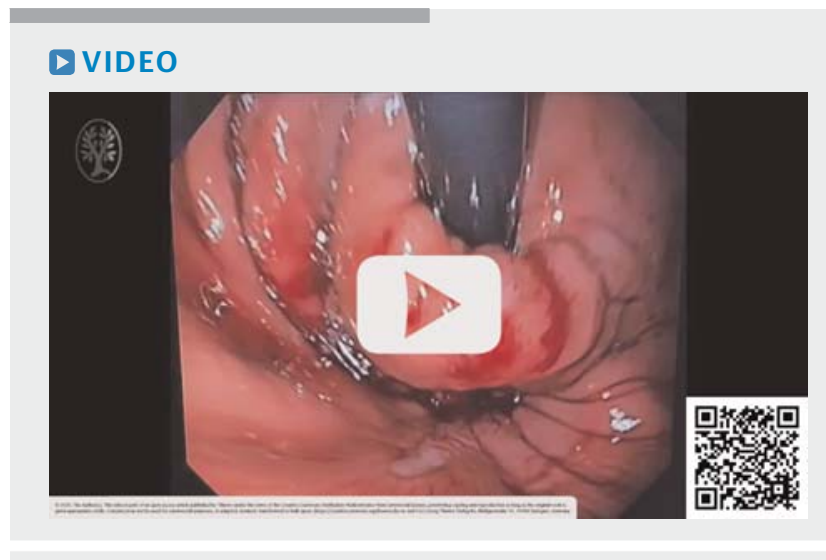

Video 1 Video of POEM-TIF in a canine model.

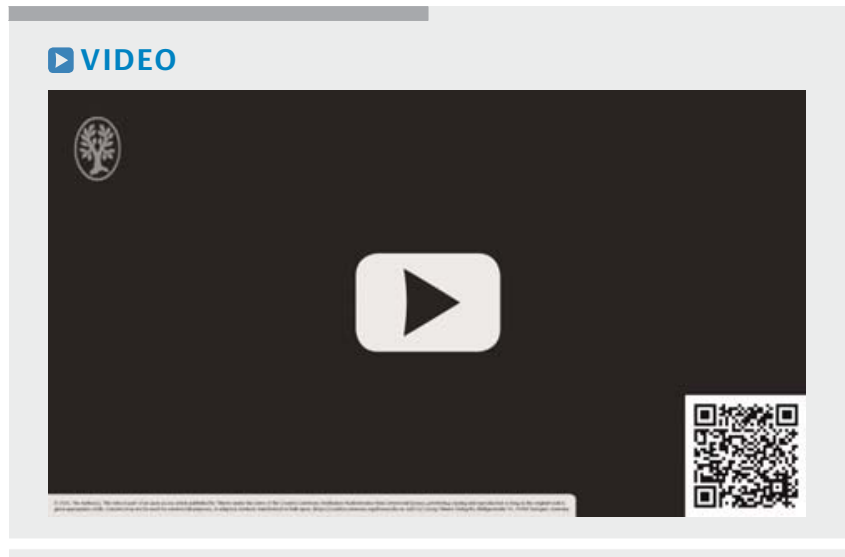

- Video2 Video of POEM-TIF in humans. sure, the mucosotomy and tunnel were inspected for trauma, bleeding and visible fasteners ( $\triangleright$ Fig. $\mathbf{2 f}$, $\triangleright$ Video $\mathbf{1}$ ). The mucosal entry was then closed with hemostatic clips (Resolution 360, Boston Scientific, Marlborough, Massachusetts, United States).

\section{Ex vivo leak testing}

A necropsy was performed, with en bloc resection of the esophagogastroduodenal specimen. The duodenum was sealed with a large vascular clamp, and the total specimen was filled with $400 \mathrm{~mL}$ of concentrated methylene blue/sterile water (2.5\% methylene blue) to achieve maximum distention. The esophagus was similarly clamped, and the entire specimen was tested for leaks in a clean water basin for a duration of two hours ( Fig.4).

\section{First-in human POEM-TIF cases}

After successful implementation of both POEM and TIF as a single combined procedure in a canine model, POEM-TIF was offered to patients as a strategy to mitigate their post-POEM GERD. The primary outcome of this study was to assess technical success of POEM and TIF performed sequentially in the same session. Secondary outcomes were to assess the improvement in dysphagia, the creation of an anatomic flap valve with the TIF, and the rate of GERD and/or erosive esophagitis. Data was reviewed retrospectively under a local IRB at each performing institution (Northwell Health and Johns Hopkins medical Center).

First, a POEM was performed as per the operator's standard technique, using either an anterior $(n=3)$ or posterior $(n=2)$ right sided approach. It was up to the operator's discretion on whether to defer closure of the mucosotomy tunnel until after completion of the TIF or immediately post POEM. The TIF was performed as described above for the canine pilot study, taking the axis of the submucosal tunnel as the 12 o'clock reference and adjusting the position of the fasteners accordingly. TIF was not performed if there were any intraprocedural adverse events such significant bleeding during the POEM, or an inadvertent mucosal injury. The tunnel was examined for fasteners, mucosal injury and bleeding if the mucosotomy closure had been deferred, and final closure was performed at the end of the procedure with either TTS hemostatic clips or endoscopic suturing
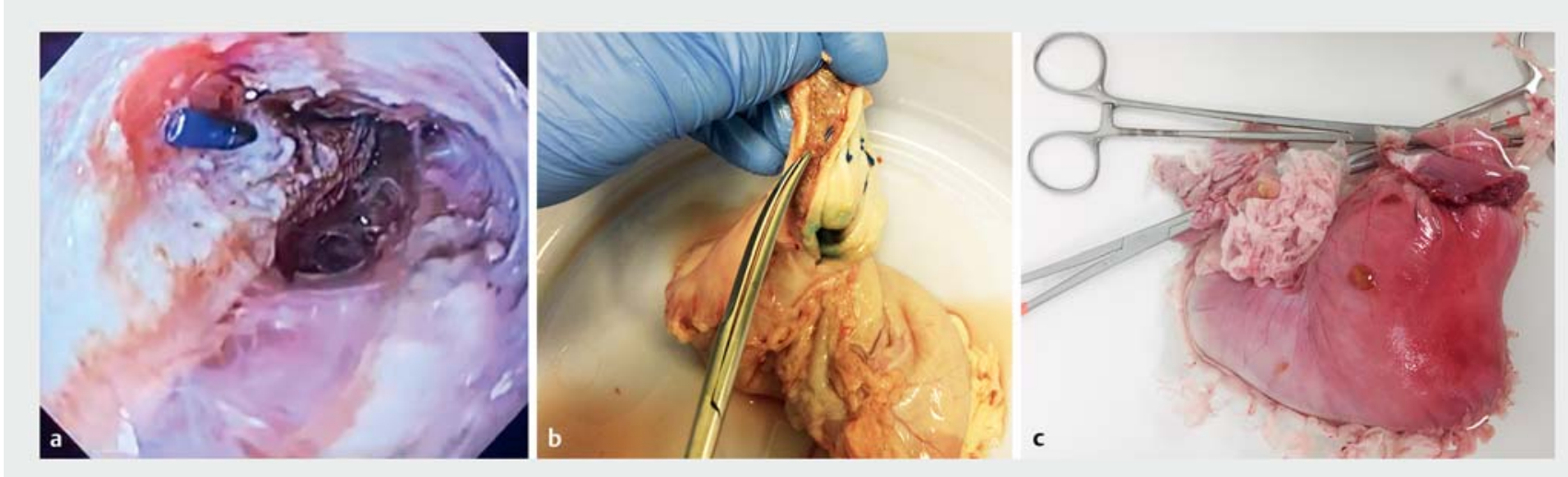

> Fig. 4 Final inspection of TIF tunnel and subsequent leak testing. After TIF is performed, we are able to inspect the mucosotomy and tunnel for damage. a In one case, a single TIF fastener is noted to have penetrated through the myotomy edge. $\mathbf{b}$ This is also demonstrated on necropsy. Extensive leak testing was performed by filling the esophagogastroduodenal specimens with concentrated methylene blue and observing them for leak in a clean water basin (c). 
- Table 1 POEM-TIF canine procedural characteristics and clinical outcomes.

\begin{tabular}{|c|c|c|c|c|c|c|}
\hline Animal Characteristics & 1 & 2 & 3 & 4 & 5 & 6 \\
\hline Species & Hound mix & Hound mix & Hound mix & Hound mix & Hound mix & Hound mix \\
\hline Weight & 21.4 & 20.3 & 21.8 & 20.1 & 22 & 21.9 \\
\hline Sex & Female & Female & Female & Female & Female & Female \\
\hline \multicolumn{7}{|l|}{ Procedural characteristics } \\
\hline Orientation of POEM & Anterior & Anterior & Posterior & Anterior & Posterior & Posterior \\
\hline POEM total time (min) & 25 & 31 & 90 & 29 & 71 & 53 \\
\hline TIF total time (min) & 21 & 22 & 26 & 15 & 19 & 20 \\
\hline $\begin{array}{l}\text { Length of myotomy (Esophagus/ } \\
\text { gastric) }(\mathrm{cm})\end{array}$ & $4 / 2$ & $5 / 2$ & $6 / 2$ & $5 / 2$ & $6 / 2$ & $5 / 3$ \\
\hline Length of POEM tunnel $(\mathrm{cm})$ & 10 & 14 & 15 & 14 & 14 & 15 \\
\hline Degree of fundoplication & 220 & 240 & 240 & 220 & 240 & 220 \\
\hline Type of myotomy & Full thickness & Full thickness & Full thickness & Full thickness & Full thickness & Full thickness \\
\hline Length of final valve $(\mathrm{cm})$ & $4 \mathrm{~cm}$ & 4 & 4 & 4 & 3 & 3 \\
\hline Reduction of mucosal entry (cm) & $5 \mathrm{~cm}$ & 4 & 5 & 4 & 3 & 2 \\
\hline Number of fasteners & 22 & 24 & 22 & 22 & 22 & 20 \\
\hline \multicolumn{7}{|l|}{ Outcomes } \\
\hline Survival & Yes & Yes & Yes & Yes & Yes & Yes \\
\hline Adverse events & PTX & None & None & None & None & None \\
\hline Injury to mucosal entry & None & None & None & None & None & None \\
\hline Injury to POEM tunnel & None & None & 1 Visible fastener & None & None & None \\
\hline Leak & None & None & None & None & None & None \\
\hline
\end{tabular}

- Video 2. Patients were admitted for overnight observation, followed by next day esophagram to exclude a leak. As per our protocol, patients were then discharged on clear liquids for 72 hours, followed by full liquids for 72 hours. The diet was then advanced according to a standard post TIF regimen. This consisted of full liquids for 7 days, followed by a soft diet at day 7 and regular diet after 14 to 21 days. Patients also completed a course of empiric antibiotics for one week and PPIs were continued for 30 days. Patient was seen in clinic 2 to 4 weeks postprocedure to assess symptoms and possible AEs. Clinical follow-up consisted of completion of GERD-HRQL questionnaires and Eckardt scores at $0,1,3$, and 6 months with a 48 -hour wireless $\mathrm{pH}$ capsule study performed at approximately 6 months off of PPIs. GERD was defined as the presence of reflux esophagitis on follow up endoscopy, an elevated DeMeester score ( $>14.72)$ or $>6 \%$ total acid exposure on either day of $\mathrm{pH}$ testing. Symptoms were assessed by GERD-HRQL as a subjective measure at 1, 3, 6 months.

\section{Results}

\section{Canine pilot study for single combined session POEM-TIF}

POEM-TIF was performed successfully in all six animals ( 3 anterior and 3 posterior POEMs) ( $\vee$ Table 1 ). There were no mucosal injuries or significant bleeding. There was one pneumothorax as result of having to use routine air insufflation for the first case, which resulted in some ventilatory instability ( $\mathrm{O} 2$ saturation, 84-94). Despite this, TIF was completed without hemodynamic instability.

The first case taught us that TIF could lengthen the esophagus, reducing the GEJ below the diaphragm. Lengthening the esophagus also transposed the mucosotomy distally by approximately the same length as the new valve ( $\bullet \mathbf{F i g} . \mathbf{5})$. The final distance between the mucosotomy and fasteners in this case was less than ideal, so we increased the length of the tunnel by $5 \mathrm{~cm}$ for subsequent cases.

Overall, the total POEM procedural time was $49.8 \pm 26.4 \mathrm{~min}$ (range $25-90 \mathrm{~min}$ ) including closure. All of the TIFs were performed on average in $20.5 \pm 3.6 \mathrm{~min}$ (range $15-26 \mathrm{~min}$ ), essentially taking up approximately one quarter of the total proce- 


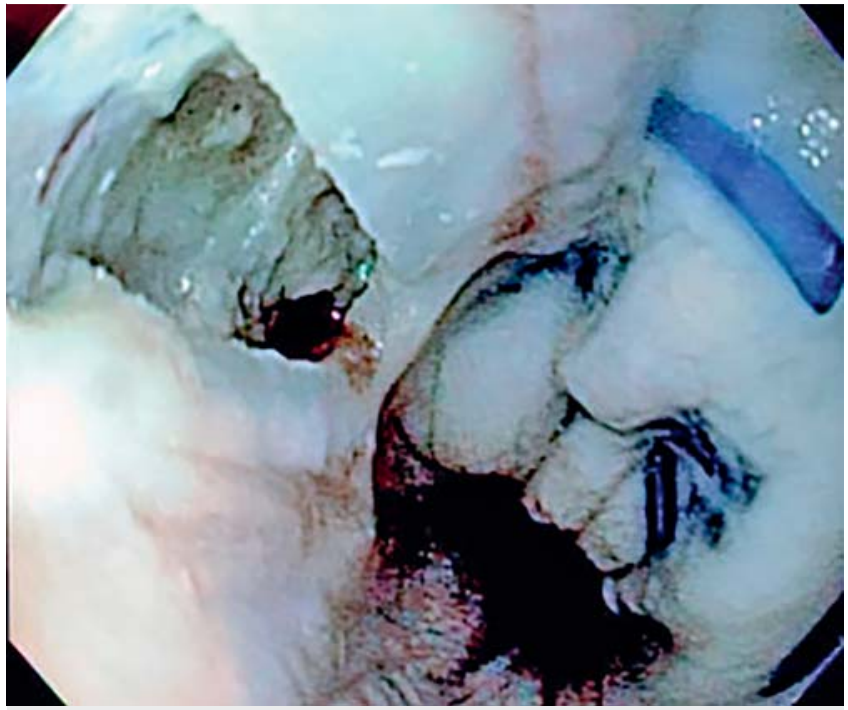

- Fig. 5 TIF results in lengthening of the esophagus. After TIF was performed on the first animal, it was evident that it had the ability to lengthen the esophagus, thereby reducing it below the diaphragm as the valve is created. In the first case it was noted that the mucosotomy was drawn distally and that we would have to compensate for this effect on subsequent cases.

dure time and with less variability. The average total procedure time was $70.3 \mathrm{~min}$ (range $44-116 \mathrm{~min}$ ).

TIF was completed in all animals with at least 20 fasteners (range 20-24) placed in a standard fashion but using the submucosal tunnel 12 O'clock reference position. The orientation of the POEM, whether anterior or posterior right, did not affect the ability to successfully perform a TIF. The degree of fundoplication was estimated during endoscopy and final necropsy and found to range from 220-240 degrees. A robust flap valve was created in all animals ranging from 3 to $4 \mathrm{~cm}$ (median $4 \mathrm{~cm}$ ) with a concomitant reduction of the esophagus and mucosal entry (range $2-5 \mathrm{~cm}$, median $4 \mathrm{~cm}$ ). Anecdotally, it seemed that performing a myotomy allowed operators to readily lengthen and wrap the GEJ beyond what is typical for this model.

Finally, a 2-hour methylene blue leak test was performed to exclude leaks. There were no intraprocedural mortalities, and save for the first case, there were no other episodes of ventilatory instability. TIF did not cause any injury to the POEM entry or tunnel. In five of six cases, the tunnel was avoided completely when placing TIF fasteners. In one case, a single fastener was observed in the tunnel, which had no effect on final leak testing.

\section{First-in-human POEM-TIF cases}

POEM-TIF was offered to patients who had a confirmed diagnosis of Achalasia and were considered candidates for POEM ( $\triangleright$ Table 2). This case series represents the results of 5 consecutive patients ( 4 women, 1 man, average age 61.2 years). Four of the patients had type II achalasia and one had type I, with an average duration of disease of 12.2 months (range 8-18 months).
For each patient, the procedure began with a POEM as per the operator's standard practice (posterior right $n=2$, posterior left $n=3)$. An esophageal full thickness myotomy was performed taking into consideration the patient's high resolution manometry results (median $10 \mathrm{~cm}$, range $5-12 \mathrm{~cm}$ ), along with a full thickness gastric myotomy as per the operator's standard practice (median $3 \mathrm{~cm}$, range $2-3 \mathrm{~cm}$ ). In all cases there was no evidence of bleeding or injury to the overlying mucosa, and so we were able to safely proceed with a TIF.

TIF was able to be performed equally well in patients that had undergone either a posterior or anterior approach by reorienting the reference point as described above. A median of 24 fasteners (range 24-29) were placed according to the POEM-TIF protocol, resulting in an average valve of $3 \mathrm{~cm}$. The addition of TIF to the POEM procedure added on average $38 \mathrm{~min}$ (range 25$50 \mathrm{~min}$ ). The tunnel was able to be examined in all cases before closure, and there was no evidence of mucosal injury or bleeding as a result of the TIF. There was evidence of a single fastener in three cases which had no clinical consequence such as a detrimental mucosal injury.

In all cases a POEM-TIF was able to be successfully performed without any intraprocedural adverse events such as bleeding, or mucosal injury. There were no AEs at 48 hours, 2 weeks and or up to 30 days thereafter. At baseline, the median Eckardt score was 7 (range 6-9) and at 6 months the median Eckardt was 2 (range $0-3$ ). Four of five patients had no evidence of GERD on 48-hour $\mathrm{pH}$ testing which correlated well with their GERD-HRQL ( Table 2). Of these four, however, one case had chronic ongoing GERD-related symptoms and required that her $\mathrm{pH}$ test be performed on twice-daily PPI, resulting in a total DeMeester of 1.5 but with evidence of esophagitis on endoscopy and so overall the exam was considered positive for GERD.

\section{Discussion}

While POEM has become a first line treatment for achalasia, long-term GERD and esophagitis threaten to diminish its success. The need for subsequent GERD procedures, surgical or endoscopic, may negate its current advantages. Recently, Inoue et al. reported on a pure natural orifice endo surgical procedure they termed POEM-Fundoplication (POEM-F); however, despite its intriguing approach and potential it may not be attainable by even the most advanced operators [16]. Therefore, we sought to understand the challenges of performing POEMTIF together as a solution to the problem of GERD after POEM.

Our initial animal pilot study and first-in-human cases suggest that POEM followed by TIF in a single session is both safe and feasible. We consider a single case of pneumothorax in the animal study a consequence of lacking a dedicated CO2 insufflator for the first case. Subsequently there were no issues related to insufflation, nor was this observed in human cases. The ability to inspect the tunnel at every step and perform extensive final leak testing in our pilot study suggests that these procedures can be safely performed together. Similarly, there were no adverse events in the first human cases during or after the procedure. 
- Table2 POEM-TIF first-in-human procedural characteristics and clinical outcomes.

\begin{tabular}{|c|c|c|c|c|c|}
\hline Patient characteristics & 1 & 2 & 3 & 4 & 5 \\
\hline Gender & $\mathrm{F}$ & $\mathrm{F}$ & M & $\mathrm{F}$ & $\mathrm{F}$ \\
\hline BMI & 22.3 & 27.2 & 35.5 & 17.2 & 27.3 \\
\hline Achalasia subtype & II & II & II & 1 & ॥ \\
\hline Duration of disease & 18 & 8 & 12 & 9 & 14 \\
\hline \multicolumn{6}{|l|}{ Procedural characteristics } \\
\hline Orientation of POEM & Posterior & Posterior & Anterior & Anterior & Anterior \\
\hline POEM total time (min) & 38 & 24 & 40 & 37 & 30 \\
\hline TIF total time (min) & 33 & 25 & 50 & 40 & 42 \\
\hline Length of myotomy (esophagus/gastric) (cm) & $7 / 2$ & $5 / 2$ & $10 / 3$ & $10 / 3$ & $12 / 3$ \\
\hline Type of myotomy & Full thickness & Full thickness & Full thickness & Full thickness & Full thickness \\
\hline Length of final valve $(\mathrm{cm})$ & 3 & 4 & 3 & 3 & 2 \\
\hline Number of fasteners & 24 & 24 & 24 & 20 & 29 \\
\hline Method of closure & TTS clips & TTS clips & Suture & Suture & TTS clips \\
\hline \multicolumn{6}{|l|}{ Technical outcomes } \\
\hline Technical success & Yes & Yes & Yes & Yes & Yes \\
\hline Adverse events & None & None & None & None & None \\
\hline Injury to mucosal entry & None & None & None & None & None \\
\hline Injury to POEM tunnel mucosa & None & None & None & None & None \\
\hline Presence of bleeding & None & None & None & None & None \\
\hline \multicolumn{6}{|l|}{ Clinical outcomes } \\
\hline Eckardt baseline & 7 & 6 & 9 & 8 & 6 \\
\hline Eckardt 1 month & 1 & 2 & 2 & 0 & 3 \\
\hline Eckardt 3 months & 1 & 1 & 1 & 2 & 3 \\
\hline Eckardt 6 months & 0 & 1 & 3 & 2 & 3 \\
\hline GERD-HRQL 1 month & 6 & 6 & 15 & 3 & 18 \\
\hline GERD-HRQL 3 months & 7 & 6 & 13 & 7 & 22 \\
\hline Gerd HRQL 6 months & 4 & 7 & 16 & 16 & 24 \\
\hline 48-hr pH testing 6 months & 2.8 & 8.4 & 3.3 & 21.6 & $1.5^{1}$ \\
\hline Esophagitis & None & None & None & LA Grade A & LA Grade C \\
\hline
\end{tabular}

While it is difficult based on this limited data to speak to the long-term anti-reflux benefit of this procedure, we observed important anatomic benefits in both the pilot study and firstin-human. First, myotomy seems to facilitate TIF, allowing for a 3- to 4-cm flap valve with concomitant lengthening of the esophagus. We observed this in both the animal pilot study and human cases. Also, lengthening the esophagus provides a straightening effect which may be beneficial. In patients with achalasia, an increase in esophageal diameter is accompanied with increased intrathoracic length and tortuosity. Despite an adequate myotomy, these patients continue to have poor esophageal clearance. Therefore, straightening the esophagus may have an unforeseen benefit for these patients as it relates to esophageal emptying. More cases will have to be performed with a focus on functional studies such as timed barium esophagrams in order to show this in humans.

Overall, the benefit of performing TIF and POEM together must be balanced with the risk of injury to the overlying mucosa. The small amount of added time withstanding, the addition of TIF did not seem to add risk or make the procedure more dif- 
ficult. If the overlying mucosa has not been compromised during the POEM, the addition of TIF seems to be safe. The mucosotomy, overlying mucosa and the submucosal tunnel were essentially unaltered by TIF. Both in the pilot study and human cases, an occasional fastener was noted to penetrate the overlying tunnel mucosa, but this did not seem to have an effect on leak testing, nor clinically in patients. In all cases, the pull of the fasteners on each side of the myotomy, kept edges of the myotomy apart on final tunnel inspection. This "buttressing" effect is an important element of Toupet fundoplication, preventing re-approximation of the myotomy. TIF may have similar effects but this needs further investigation. It is not clear whether performing TIF separately will provide a similar benefit-inability to recognize orientation of the prior myotomy during TIF may artificially reapproximate edges. This may explain why anecdotally there is an increase in dysphagia for some patients receiving TIF after POEM [15]. This is an important consideration when making a decision to offer TIF and POEM in one session.

POEM-TIF does not address all the potential causes of GERD post POEM and therefore it is unlikely to eliminate the problem completely. Occasionally, some patients with achalasia also have a clinically significant but unrecognized hernia. This is difficult to ascertain during the POEM procedure and continues to remain one of the short comings of a combined procedure. A hiatal hernia in patients with achalasia has been noted in up to $20 \%$ of cases, but a truly large hernia requiring cruroplasty is only noted about $10 \%$ of the time. In considering this approach we may have to pay special attention to barium esophagrams to assess the possibility of a moderate to large hernia. In our case series, failure to identify a small hernia during the POEM-TIF explains the one patient with esophagitis and significant clinical symptoms that were difficult to resolve even on PPI.

\section{Conclusions}

In conclusion, TIF after POEM appears to be safe and feasible in a canine model, and this was also noted in our first in human experience. The motivation for combining these two procedures goes beyond the obvious anti-reflux benefit. The extra costs of TIF may be balanced by the reduction in long term GERD sequalae. This animal study offers a template for how we may approach this combined procedure in humans and the first human cases suggest that this template can be successful. Further refinements in technique along with greater patient experience will likely improve outcomes and should help us understand whether this can be a solution for the problem of GERD after POEM.

\section{Acknowledgements}

Equipment and support for the laboratory was provided by EndoGastric Solutions and ERBE. Technical support for the laboratory was provided by Adrian Lobontiu, MD
Dr. Benias is a consultant for FujiFilm, Apollo Endosurgery, and Medtronic. Dr. Kumta is a consultant for Apollo Endosurgery. Dr. Sethi is a consultant for FujiFilm and Boston Scientific. Dr. Kumbhari is a consultant for Medtronic, Pentax Medical, Boston Scientific, FujiFilm, and Apollo Endosurgery and receives research support from ERBE USA and Apollo Endosurgery. Dr. Khashab is a consultant for Olympus USA and Boston Scientific.

\section{References}

[1] Khashab MA, Benias PC, Swanstrom LL. Endoscopic myotomy for foregut motility disorders. Gastroenterology 2018; 154: 1901-1910

[2] Haito-Chavez Y, Inoue H, Beard KW et al. Comprehensive analysis of adverse events associated with per oral endoscopic myotomy in 1826 patients: An international multicenter study. Am J Gastroenterol 2017; 112: 1267-1276

[3] Kumbhari V, Familiari P, Bjerregaard NC et al. Gastroesophageal reflux after peroral endoscopic myotomy: a multicenter case-control study. Endoscopy 2017; 49: 634-642

[4] Repici A, Fuccio L, Maselli R et al. GERD after per-oral endoscopic myotomy as compared with Heller's myotomy with fundoplication: a systematic review with meta-analysis. Gastrointest Endosc 2018; 87: 934-943

[5] Campos GM, Vittinghoff E, Rabl C et al. Endoscopic and surgical treatments for achalasia: a systematic review and meta-analysis. Ann Surg 2009; 249: 45-57

[6] Rösch T, Repici A, Boeckxstaens G. Will reflux kill POEM? Endoscopy 2017; 49: 625-628

[7] Ichkhanian Y, Benias P, Khashab MA. A case of early Barrett cancer following peroral endoscopic myotomy. Gut 2019; 68: 2107-2110

[8] Richards WO, Torquati A, Holzman MD et al. Heller myotomy versus Heller myotomy with Dor fundoplication for achalasia: a prospective randomized double-blind clinical trial. Ann Surg 2004; 240: 405-412

[9] Schlottmann F, Luckett DJ, Fine J et al. Laparoscopic Heller myotomy versus peroral endoscopic myotomy (POEM) for achalasia: a systematic review and meta-analysis. Ann Surg 2018; 267: 451-460

[10] Hunter JG, Kahrilas PJ, Bell RC et al. Efficacy of transoral fundoplication vs omeprazole for treatment of regurgitation in a randomized controlled trial. Gastroenterology 2015; 148: 324-333

[11] Trad KS, Barnes WE, Simoni G et al. Transoral incisionless fundoplication effective in eliminating GERD symptoms in partial responders to proton pump inhibitor therapy at 6 months: the TEMPO randomized clinical trial. Surg Innov 2015; 22: 26-40

[12] Sami Trad K. Transoral incisionless fundoplication: current status. Curr Opin Gastroenterol 2016; 32: 338-343

[13] Testoni PA, Testoni S, Mazzoleni G et al. Long-term efficacy of transoral incisionless fundoplication with Esophyx (Tif 2.0) and factors affecting outcomes in GERD patients followed for up to 6 years: a prospective single center study. Surg Endosc 2015; 29: 2770-2780

[14] Tyberg A, Choi A, Gaidhane M et al. Transoral incisional fundoplication for reflux after peroral endoscopic myotomy: a crucial addition to our arsenal. Endosc Int Open 2018; 6: E549-E552

[15] Brewer Gutierrez OI, Benias PC, Khashab MA. Same-session per-oral endoscopic myotomy followed by transoral incisionless fundoplication in achalasia: Are we there yet? Am J Gastroenterol 2020; 115: 162

[16] Haruhiro I, Akiko U, Yuto S et al. Peroral endoscopic myotomy and fundoplication: a novel NOTES procedure. Endoscopy 2019; 51: 161164 